\title{
Tip of the red giant branch distance for the Sculptor group dwarf ESO 540-032^
}

\author{
H. Jerjen ${ }^{1}$ and M. Rejkuba ${ }^{2,3}$ \\ 1 Research School of Astronomy and Astrophysics, The Australian National University, Mt Stromlo Observatory, \\ Cotter Road, Weston ACT 2611, Australia \\ 2 European Southern Observatory, Karl-Schwarzschild-Strasse 2, 85748 Garching bei München, Germany \\ e-mail: mrejkuba@eso.org \\ 3 Department of Astronomy, P. Universidad Católica, Casilla 306, Santiago 22, Chile
}

Received 19 January 2001 / Accepted 9 March 2001

\begin{abstract}
We present the first VI CCD photometry for the Sculptor group galaxy ESO 540-032 obtained at the Very Large Telescope UT1+FORS1. The $(I, V-I)$ colour-magnitude diagram indicates that this intermediatetype dwarf galaxy is dominated by old, metal-poor $([\mathrm{Fe} / \mathrm{H}] \approx-1.7 \mathrm{dex})$ stars, with a small population of slightly more metal-rich $([\mathrm{Fe} / \mathrm{H}] \approx-1.3 \mathrm{dex})$, young (age $150-500 \mathrm{Myr}$ ) stars. A discontinuity in the $I$-band luminosity function is detected at $I_{0}=23.44 \pm 0.09 \mathrm{mag}$. Interpreting this feature as the tip of the red giant branch and adopting $M_{I}=-4.20 \pm 0.10 \mathrm{mag}$ for its absolute magnitude, we have determined a Population II distance modulus of $(m-M)_{0}=27.64 \pm 0.14 \mathrm{mag}(3.4 \pm 0.2 \mathrm{Mpc})$. This distance confirms ESO $540-032$ as a member of the nearby Sculptor group but is significantly larger than a previously reported value based on the Surface Brightness Fluctuation (SBF) method. The results from stellar population synthesis models suggest that the application of the SBF technique on dwarf galaxies with mixed morphology requires a detailed knowledge of the underlying stellar composition and thus offers no advantage over a direct distance measurement using the tip of the red giant branch as distance indicator. We produce the surface brightness profiles for ESO $540-032$ and derive the photometric and structural parameters. The global properties follow closely the relations between metallicity and both absolute magnitude and central surface brightness defined by dwarf elliptical galaxies in the Local Group. Finally, we identify and discuss a non-stellar object near the galaxy center which may resemble a globular cluster.
\end{abstract}

Key words. galaxies: abundances - galaxies: clusters: individual: Sculptor - galaxies: dwarf - galaxies: individual: ESO 540-032 - galaxies: stellar content - galaxies: structure

\section{Introduction}

Dwarf elliptical galaxies (hereafter dEs, subsuming "dwarf spheroidal galaxies") in the Local Group exhibit a rather diverse and complex set of star formation histories (Da Costa 1998; Grebel 1998). Their stellar populations range from almost exclusively old (Ursa Minor, Olszewski \& Aaronson 1985) to mainly old (e.g. Tucana, Da Costa 1998) with an observed intermediate-age episodic SFH in Carina (Mighell 1997; Hurley-Keller et al. 1998) and an intermediate-age continuous SFH in Fornax (Stetson et al. 1998). Phoenix and LSG3 are classified as intermediate-type dwarfs $(\mathrm{dE} / \mathrm{Im})$, because they show similarities to both dwarf ellipticals and dwarf irregulars. These systems are dominated by an old metal-poor

\footnotetext{
Send offprint requests to: H. Jerjen,

e-mail: jerjen@mso.anu.edu.au

* Based on observations collected at the European Southern Observatory (ESO 64.N-0069).
}

population with no evidence for major star formation activities after the initial episode 8-10 Gyr ago. However, both systems also accommodate a minor population of young stars, with ages of about 150-500 Myr, which makes these galaxies resemble dwarf irregulars (Held et al. 1999; Martínez-Delgado et al. 1999; Holtzman et al. 2000; Aparicio et al. 1997).

This rich collection of star formation histories in the Local Group led in recent years to a large effort to find and physically characterise more of these faintest, most elusive galaxies beyond the group boundary. An extensive search for dwarf galaxies in the closest galaxy aggregate to the Local Group, the Sculptor (Scl) Group at $\approx 2.5 \mathrm{Mpc}$, was carried out by Côté and collaborators (1995; Côté et al. 1997) confirming 16 gas-rich dwarf members based on pointed $\mathrm{HI}$ and $\mathrm{H} \alpha$ observations and redshift measurements. Her list was supplemented by a first set of five gas-poor $\mathrm{dE}$ and intermediate-type dwarf galaxies (Jerjen et al. 1998, hereafter JFB98; 2000b) whose distances were 
estimated with the Surface Brightness Fluctuation (SBF) method. Thereby, the zero point for the reported SBF distances had to rely on theoretical predictions of stellar population synthesis models and on assumptions about the dwarfs' stellar contents and star formation histories. The lack of further distance information initiated our work to get a distance confirmation for some of these dwarf galaxies based on their resolved stellar populations.

Among these new Scl group dwarfs was ESO 540-032, also known as KK010 (Karachentseva \& Karachentsev 1998). This galaxy was initially classified as intermediatetype dwarf, dE/Im (JFB98) or equivalent Sph/dIrr (Karachentseva \& Karachentsev 1998) based on its dElike overall smooth light distribution and the knotty structure, typical for members of the dwarf irregular family. These morphological features are visible in Fig. 1 showing $V$ and $I$ CCD images of the galaxy. ESO 540-032 remained undetected in the pointed Hi surveys by Gallagher et al. (1995), Côté et al. (1997) and Karachentsev et al. (1999). Côté et al. quote a $3 \sigma$ detection limit of $410^{6} M_{\odot} \mathrm{HI}$ mass which is a factor of 10 above the expected H I mass for other such dwarf systems (St-Germain et al. 1999).

In this paper we present the first VI CCD photometry of the stellar content of the Scl group dwarf ESO 540-032. In Sect. 2 we describe the observations, data reduction, and the photometry completeness tests. In Sect. 3 we analyse the resulting colour-magnitude diagram. The tip magnitude of the red giant branch is measured and the distance of the galaxy determined based on this method in Sect. 4. The latter result is compared with the SBF distance in Sect. 5. Finally, surface brightness profiles and parameters from Sérsic model fitting are derived in Sect. 6 and a globular cluster candidate discussed in Sect. 7 .

\section{Observations and reductions}

Deep CCD images of ESO 540-032 were acquired in service mode using FORS1 (FOcal Reducer and low dispersion Spectrograph) at Antu (UT1) VLT at ESO Paranal Observatory. The FORS1 detector is a $2048 \times 2048$ Tektronix CCD, thinned and anti-reflection coated. The pixel size is $24 \times 24 \mu \mathrm{m}$. The field of view was $6.8 \times 6{ }^{\prime} 8$ and the pixel scale 0 !' 2 / pixel.

Three $660 \mathrm{~s}$ exposures were taken in the $V$-band during the night of 1999 November 13, and three $550 \mathrm{~s} I$-band exposures were secured during the night of 2000 January 7. The summary of observations specifying air masses and seeing conditions is given in Table 1. Photometric standard stars from the lists of Landolt (1992) and Walker (1995) were observed in both $V$ and $I$ filters, during the first and the second night, respectively.

In service mode, direct images are read out by default in the four-port mode, i.e. four amplifiers read out one quadrant of the CCD each. Xccdred, an $\mathrm{IRAF}^{1}$ package

\footnotetext{
${ }^{1}$ IRAF is distributed by the National Optical Astronomy Observatories, which is operated by the Association of
}

specially developed to reduce such images, was used to subtract overscan for each amplifier area individually and later to subtract the bias and flat-field the images. The three images in each filter were then registered using $i m a-$ lign and averaged with imcombine. Finally, the crreject algorithm was employed to reject cosmic rays.

\subsection{Photometric calibrations}

In service mode at Paranal, a set of photometric standard stars from the Landolt (1992) catalogue are observed in various passbands each night. On November 13, two Landolt fields, totalling 12 stars were observed and on January 7, four Landolt fields, totalling 19 stars. In order to have more standards that cover a broader range in colour and in brightness, we further observed a standard star sequence close to NGC 300 that was established by Graham (1981) and Walker (1995). In this way a total of 28 and 35 standards could be used to derive extinction coefficients and zero points of the two nights separately. The derived photometric transformations show that the two nights had different zero points. Since our scientific observations in $V$ and $I$-band were taken on different nights, we calibrated the data in two steps.

First, the zero point and extinction coefficients were calculated for each night separately using the equation:

$m_{\text {inst }}=\mathrm{ZP}+k \times$ Airmass $+m_{\text {cat }}$

where $m_{\text {inst }}$ and $m_{\text {cat }}$ are the instrumental and catalogued magnitudes, $\mathrm{ZP}$ is the zero point and $k$ is the extinction coefficient. We defined magnitudes that were calibrated with this zero order correction for atmospheric effects as $m_{\text {fit }}=m_{\text {inst }}-\mathrm{ZP}-k \times$ Airmass.

After we determined the zero point and extinction coefficient, the dependence of the calibration on the colour term was explored. It turned out that the colour term was important for the $V$-band calibration but negligible for the $I$-band. This allowed to put $I_{\text {real }}=I_{\text {fit }}$ (see also the value of colour coefficient $c$ in Table 2).

The second step in calibration involved the equation of the form for $V$-band:

$V_{\text {real }}-V_{\text {fit }}=c \times(V-I)_{\text {fit }}+b$

where $V_{\text {real }}$ is the magnitude from the standard star catalogue, $V_{\text {fit }}$ is the magnitude computed after the zero point and extinction corrections (Eq. (1)), $c$ is the colour coefficient and $b$ is the constant of the linear fit. The colour term depends on telescope and detector characteristics and should be constant in time. We computed the colour term for $V$-band for both nights separately and with the combined data and the three values are almost identical and well within the errors of the fit. The values of $\mathrm{ZP}, k$, $c$ and $b$ for $V$-band and $I$-band calibration are reported in Table 2. We note that the given $c$ and $b$ values are mean values for the two nights.

Universities for Research in Astronomy, Inc., under contract with the National Science Foundation. 

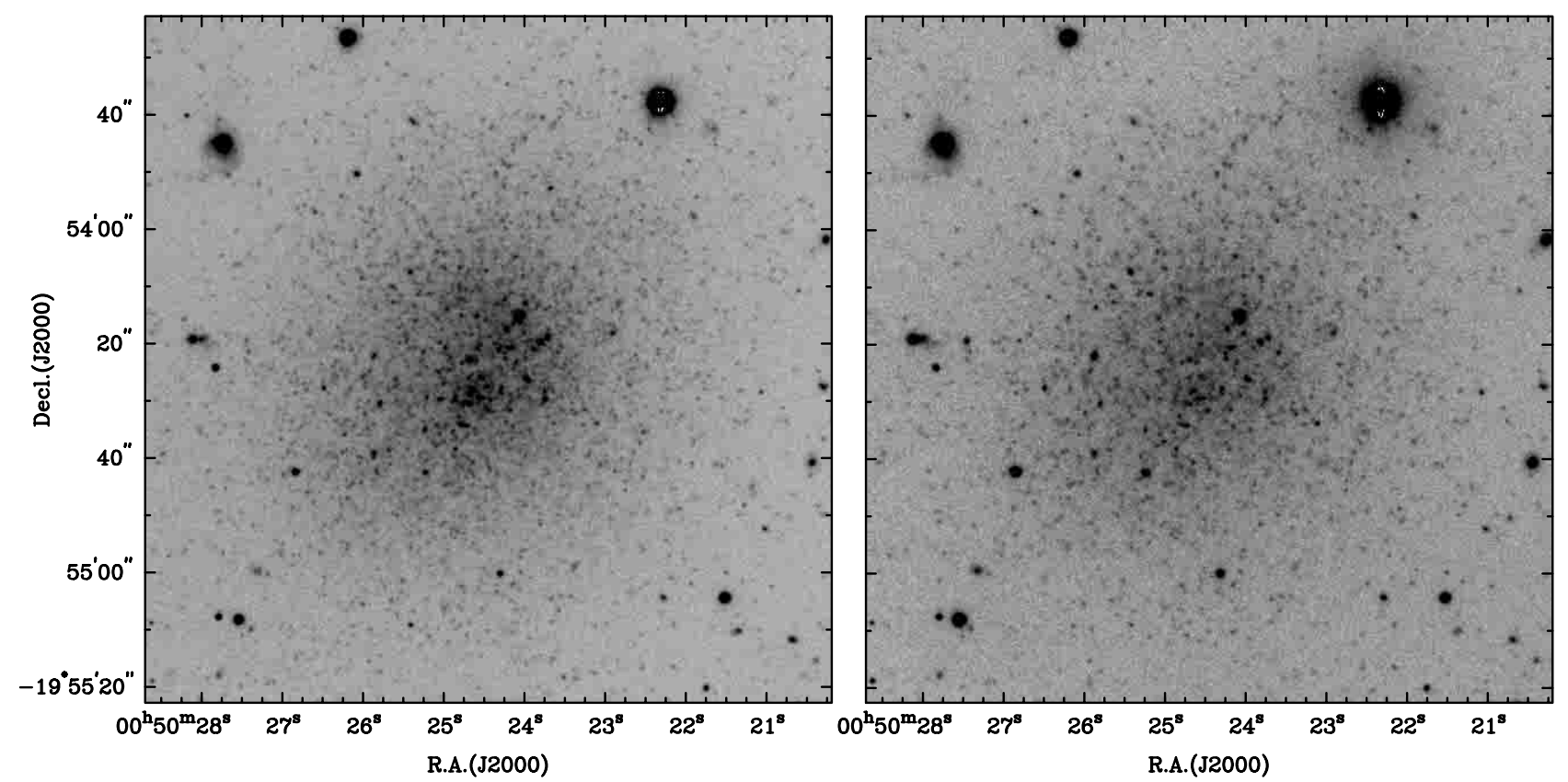

Fig. 1. $V$ (left) and $I$-band (right) images of ESO 540-032, taken at the VLT (UT1+FORS1) and showing resolution into stars. The field is $3^{\prime} .5 \times 33^{\prime} .5$. North is at the top and east to the left

Table 1. Summary of observations

\begin{tabular}{|c|c|c|c|c|c|c|c|}
\hline $\begin{array}{c}\text { RA (J2000) } \\
(\mathrm{h} \min \mathrm{s})\end{array}$ & $\begin{array}{c}\text { Decl. (J2000) } \\
\left({ }^{\circ},{ }^{\prime \prime}\right)\end{array}$ & $\begin{array}{c}\text { Date } \\
(\mathrm{dd} / \mathrm{mm} / \mathrm{yy})\end{array}$ & $\begin{array}{l}\text { Telescope \& } \\
\text { Instrument }\end{array}$ & $\begin{array}{c}\text { Exposure } \\
(\mathrm{s})\end{array}$ & Filter & Airmass & $\begin{array}{c}\text { Seeing } \\
\left({ }^{\prime \prime}\right)\end{array}$ \\
\hline 005018 & -195545 & $13 / 11 / 2000$ & Antu+FORS1 & $3 \times 660$ & $V$ & 1.25 & 0.56 \\
\hline 005018 & -195545 & $07 / 01 / 2000$ & Antu+FORS1 & $3 \times 550$ & $I$ & 1.20 & 0.67 \\
\hline
\end{tabular}

Table 2. Photometric calibration coefficients

\begin{tabular}{cccccccccc}
\hline \hline Night & Filter & ZP & $\sigma_{\mathrm{ZP}}$ & $k$ & $\sigma_{\mathrm{k}}$ & $c$ & $\sigma_{\mathrm{c}}$ & $b$ & $\sigma_{\mathrm{b}}$ \\
\hline \hline 1999 Nov. 13 & $V$ & -27.276 & 0.07 & 0.105 & 0.06 & 0.077 & 0.013 & -0.041 & 0.013 \\
2000 Jan. 07 & $I$ & -26.292 & 0.07 & 0.138 & 0.06 & -0.007 & 0.015 & 0.009 & 0.015 \\
\hline
\end{tabular}

Applying the transformations described above reproduce the standard magnitudes with a rms of 0.04 in $V$ and 0.05 in $I$. Figure 2 shows the difference between the real and calibrated magnitudes of standard stars as a function of magnitude and colour.

The photometric measurements were performed within the IRAF environment using DAOPHOT II (Stetson 1987). All the objects in the field with more than $3 \sigma$ above the background level were located separately in the $V$ and $I$ frames. A variable point-spread function (PSF) was constructed by using 32 and 33 stars in the $V$ and $I$ frames, respectively. The magnitudes were then measured using the allstar task. Only objects that matched in both $V$ and $I$ band with good photometry (i.e. $\sigma_{\text {mag }}<0.5,-1<\operatorname{sharp}<1$ and $\operatorname{chi}<2$ ) were retained. Restricting the sharp and chi parameters excludes extended objects like galaxies and stellar blends as well as any remaining blemishes. The resulting $(V, V-I)$ and $(I, V-I)$ colour-magnitude diagrams consisting of all 1830 stars detected on the CCD are shown in Fig. 3.

\subsection{Completeness and contamination}

We made extensive tests to measure completeness and magnitude errors as function of magnitude and radial distance from the center of the galaxy. Using the addstar routine in DAOPHOT II (Stetson 1987) we added 400 stars at a time to $V$ and $I$ frame, all with the same magnitude and uniformly distributed over the whole field, and re-computed the photometry. This operation was repeated for 5 different positions of added stars and at each 0.5 magnitudes in order to have a statistically significant sample of 2000 added stars per magnitude bin. Incompleteness, defined as a recovery rate of $50 \%$ for the stars from the artificial-star experiment, sets in at $V=26.1$ and $I=24.3$. The latter value is well below the magnitude of the tip of the red giant branch, i.e. $I \approx 23.5$ (see Sect. 4 ) 

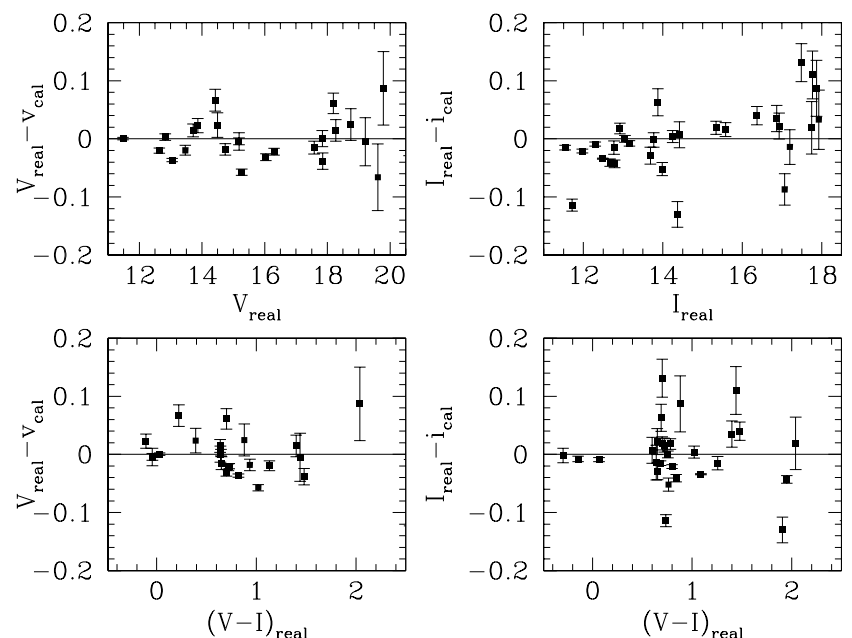

Fig. 2. Difference between the real and observed (calibrated) magnitudes of standard stars as a function of magnitude (upper two panels) and colour (bottom panels). Error bars represent combined errors of photometry and errors of the catalogued magnitudes

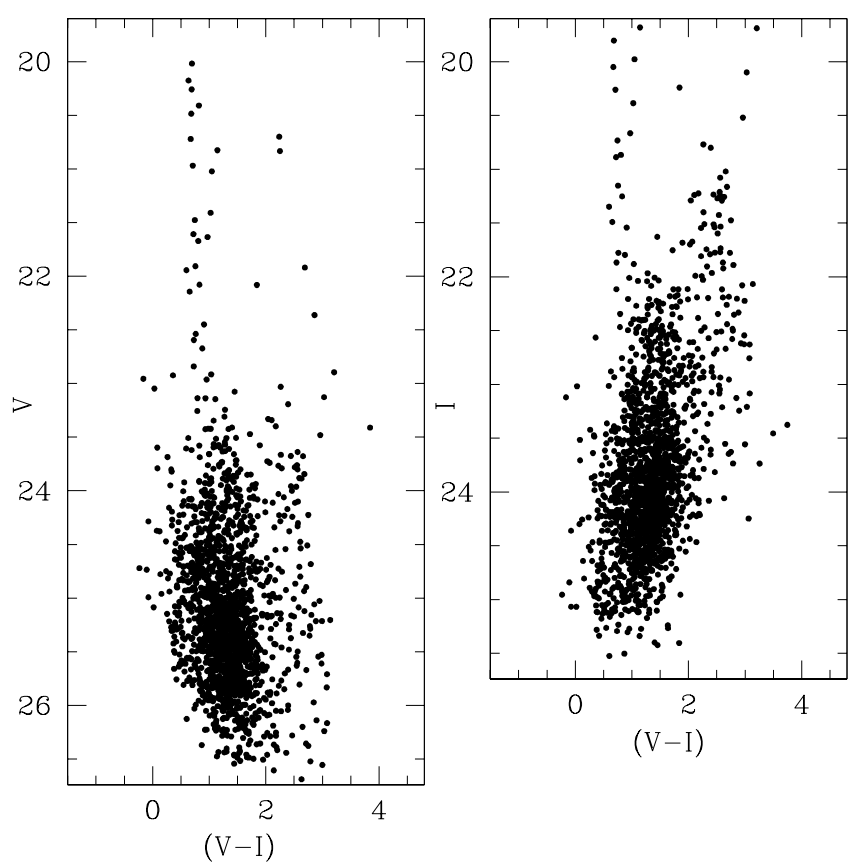

Fig. 3. Colour-magnitude diagrams of all 1830 stars matched in the $V$ and $I$-band CCD images

at which level our photometry is complete more than $90 \%$ (Fig. 4).

The artificial-star tests were performed also to assess the accuracy of our photometry. The difference between the recovered and input magnitude as a function of magnitude and as a function of radial distance from the galaxy center are shown on the right panels of Figs. 4 and 5 . From these experiments we conclude that our photometry is reliable down to the incompleteness limit. The measured magnitudes of fainter stars are systematically brighter.
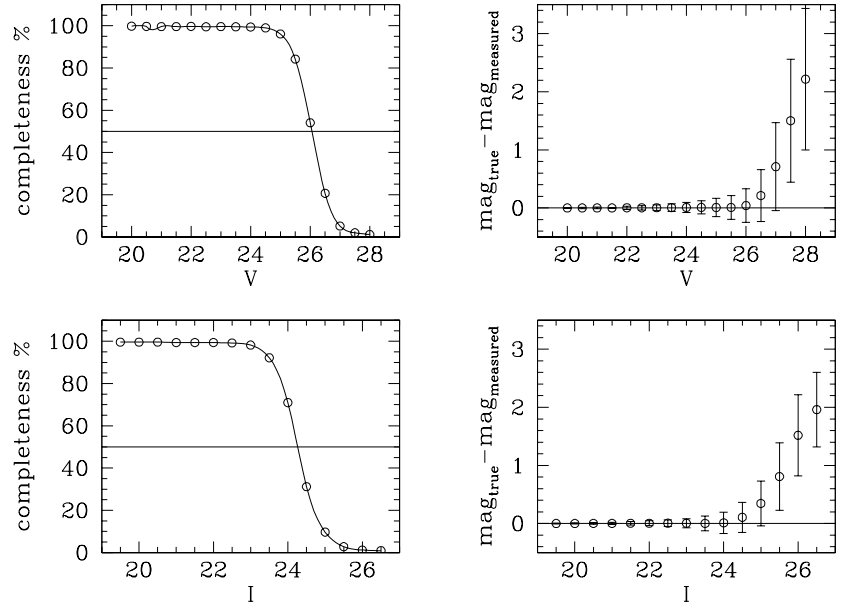

Fig. 4. Artificial-star tests: completeness as function of magnitude (left panels) and photometric accuracy as a function of magnitude (right panels) for the $V$ and $I$ band
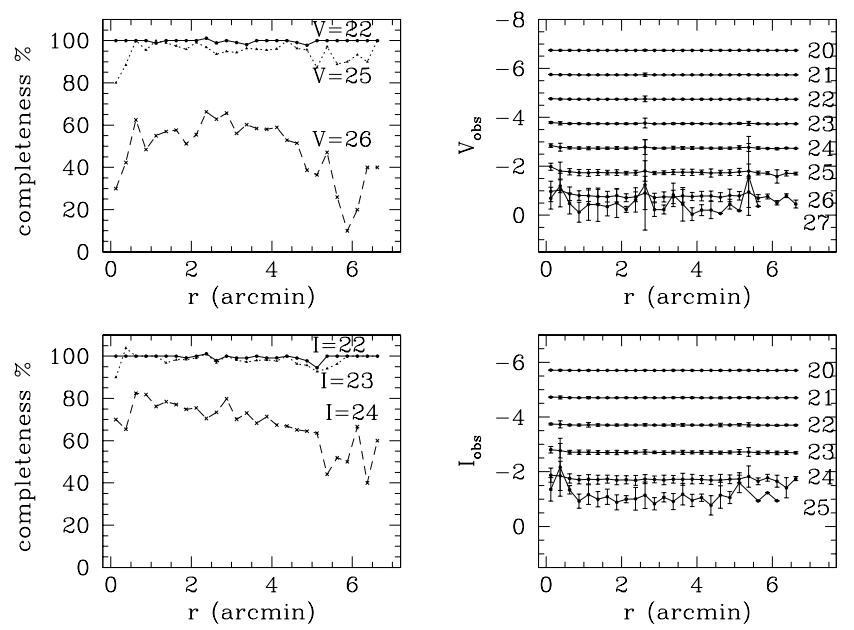

Fig. 5. Artificial-star tests: completeness and photometric accuracy as function of radial distance from the center of ESO 540-032. On the right panel $Y$-axis is the instrumental observed magnitude, while the label indicates the corresponding calibrated magnitudes

On the left panels of Fig. 5 the completeness as function of radial distance is shown for three different input magnitudes, at 100, 90 and $50 \%$ completeness levels. For brighter magnitudes there is no radial dependence on completeness, nor systematic differences in input and recovered magnitudes (right panels), but for magnitudes close to $90 \%$ completeness level the inner most bin, the circle within the 0.125 arcmin from the galaxy center, has significantly lower completeness and the recovered magnitudes start to be systematically brighter because of blending effects due to crowding. Blending with other stars results in brightening of the measured magnitudes by $\sim 0.1$ mag at the level of $90 \%$ completeness and only for the inner most radial bin. 
There is only little foreground contamination from the Galactic halo and disk due to low galactic latitude of the galaxy $\left(l=121.01^{\circ}, b=-82.77^{\circ}\right)$. Most of the foreground stars appear as a plume of bright blue stars around $V-I \sim 0.7$ (see Fig. 3). We used the Besancón group model of stellar population synthesis of the Galaxy (Robin et al. 1996) to estimate the total number of galactic foreground stars in our field. They amount to 84 stars in our observed magnitude range $(20<V<26.5)$, and $1 / 3$ of them are mixed with ESO 540-032 stars. However, we are interested in the restricted magnitude range around the red giant branch (RGB) tip where only few foreground stars could be found.

Most of the background galaxies are extended and rejected by the sharp and chi parameter constrains in our photometry, but some compact galaxies might still contaminate the sample. Since the optical size of ESO 540032 is much smaller than the FORS1 field of view, we centered the galaxy on the upper left quadrant. In that way we can also estimate the foreground stellar and background galaxy contamination by counting the number of objects in our final photometry list that are detected in the lower right corner of the CCD. There are 121 objects in the area corresponding to $\approx 1 / 4$ of CCD. Some of them belong to the halo of ESO 540-032, but mostly they are galactic stars and compact background galaxies.

\section{Colour-magnitude diagram and stellar contents}

The central panel of Fig. 6 shows the $(I, V-I)$ colourmagnitude diagram (CMD) produced from all 469 stars identified within a 40 arcsec radius from the galaxy center, an area which covers the main body of ESO 540032. The most prominent feature is a well-defined red giant branch with an approximate tip magnitude $I \approx$ 23.5. The colour dispersion of the RGB stars suggests a wide range in metallicity. Superimposed on the CMD are RGB fiducials from Da Costa \& Armandroff (1990) for the Galactic globular clusters M $15([\mathrm{Fe} / \mathrm{H}]=-2.17)$, M $2([\mathrm{Fe} / \mathrm{H}]=-1.58)$, NGC $1851(\mathrm{Fe} / \mathrm{H}]=-1.16)$, and $47 \mathrm{Tuc}([\mathrm{Fe} / \mathrm{H}]=-0.71)$. The giant branches are reddened by $E(B-V)=0.020 \mathrm{mag}$ (Schlegel et al. 1998) and brought to the distance of ESO 540-032 using the distance modulus of $27.68 \mathrm{mag}$ (see Sect. 4) in order to match the observed CMD. The majority of stars are metal-poor with $-2.17<[\mathrm{Fe} / \mathrm{H}]<-1.16$. There are also a few stars as metal-rich as 47 Tuc but almost none are more metal-rich. Caldwell et al. (1998) presented a quadratic relation between the mean metallicity and the dereddened giant branch colour $(V-I)_{0,-3.5}$ at $M_{I}=-3.5:\langle[\mathrm{Fe} / \mathrm{H}]\rangle=-1.00+1.97 q-3.2 q^{2}$, where $q=\left[(V-I)_{0,-3.5}-1.6\right]$. Using this equation with the median colour $(V-I)_{0,-3.5}=1.34 \pm 0.04$ for stars in the magnitude interval $24.13<I<24.23$ (corresponding to $M_{I}=-3.5 \pm 0.05$ for ESO 540-032), we derive $\langle[\mathrm{Fe} / \mathrm{H}]\rangle=-1.7( \pm 0.3)$ dex where the given error includes uncertainties in the distance modulus, photometry, reddening, and a rms of 0.08 dex from the relation.
A noticeable number of stars are brighter than the tip magnitude. They could be intermediate-age AGB stars. However, CMDs of two comparison fields on the CCD with the same area as the galaxy field and situated $\approx 4$ arcmin west and south of the galaxy center (left and right panel of Fig. 6) show a similar amount of foreground stars in the critical magnitude range $22<I<23.5$. This field contamination prevents a detailed discussion of the existence or a number estimate of AGB stars in ESO 540-032 with the current photometric data.

The nature of ESO 540-032 being an intermediate-type dwarf galaxy is revealed by the presence of a small population of blue stars. The panels in Fig. 6 illustrate that stars bluer than $(V-I) \approx 0.6$ are found most exclusively in the galaxy area (only one such star appears in each comparison field) thus a confusion by foreground stars can be ruled out. These stars are likely to be the bluest extent of the He burning phase for metal-poor stars with $-1.7 \leq[\mathrm{Fe} / \mathrm{H}] \leq-1.3$ and ages in the range $150-500 \mathrm{Myr}$ (see Fig. 13 of Martínez-Delgado et al. 1999). Overall, the CMD of ESO 540-032, as far as it has been recovered ( $\approx 1.5$ mag below the RGB tip), has features in common with the CMDs of the intermediate-type dwarfs Phoenix (Held et al. 1999; Martínez-Delgado et al. 1999; Holtzman et al. 2000) and LSG3 (Aparicio et al. 1997).

Finally, we look at the distribution of the stars in the galaxy (Fig. 7). All stars bluer than $(V-I)=0.9$ appear strongly concentrated in the galaxy center with a rapid decline in numbers to larger radii. A less steep gradient is found for the red stars which is consistent with the results from the surface brightness profiles of ESO 540-032 (see Sect. 5 and Jerjen et al. 2000b) where the half light radius is measured to be significantly smaller in $B$ than in $I\left(r_{B, \mathrm{eff}}=22.6 \pm 0.9 \operatorname{arcsec}\right.$ versus $\left.r_{I, \mathrm{eff}}=29.4 \mathrm{arcsec}\right)$. The difference in the stellar distributions suggest the most recent star formation activity took place close to the center of the galaxy.

\section{Tip of the Red Giant Branch Distance}

The tip magnitude of the red giant branch (TRGB) which marks the core helium flash of low-mass Population II stars has been proven to be a reliable and accurate distance indicator. Original work on the empirical calibration of the method was based on observations of giant branches for standard Galactic globular clusters (Da Costa \& Armandroff 1990). A more recent study by Gratton et al. (1997) used the Hipparcos subdwarf parallax distance scale to recalibrate these data. The absolute magnitude of the tip in the $I$-band was found to be virtually constant at $M_{I}=-4.2 \mathrm{mag}$ for ages $2-15 \mathrm{Gyr}$ and for metallicities between $-2.2<[\mathrm{Fe} / \mathrm{H}]<-0.7$ with a scatter of only $\pm 0.1 \mathrm{mag}$. These empirical results are well supported by the theoretical RGB models of Salaris \& Cassisi (1998).

Since the TRGB method works for old and metalpoor stellar populations, we can use the well-defined RGB branch in the CMD of ESO 540-032 to measure the galaxy 


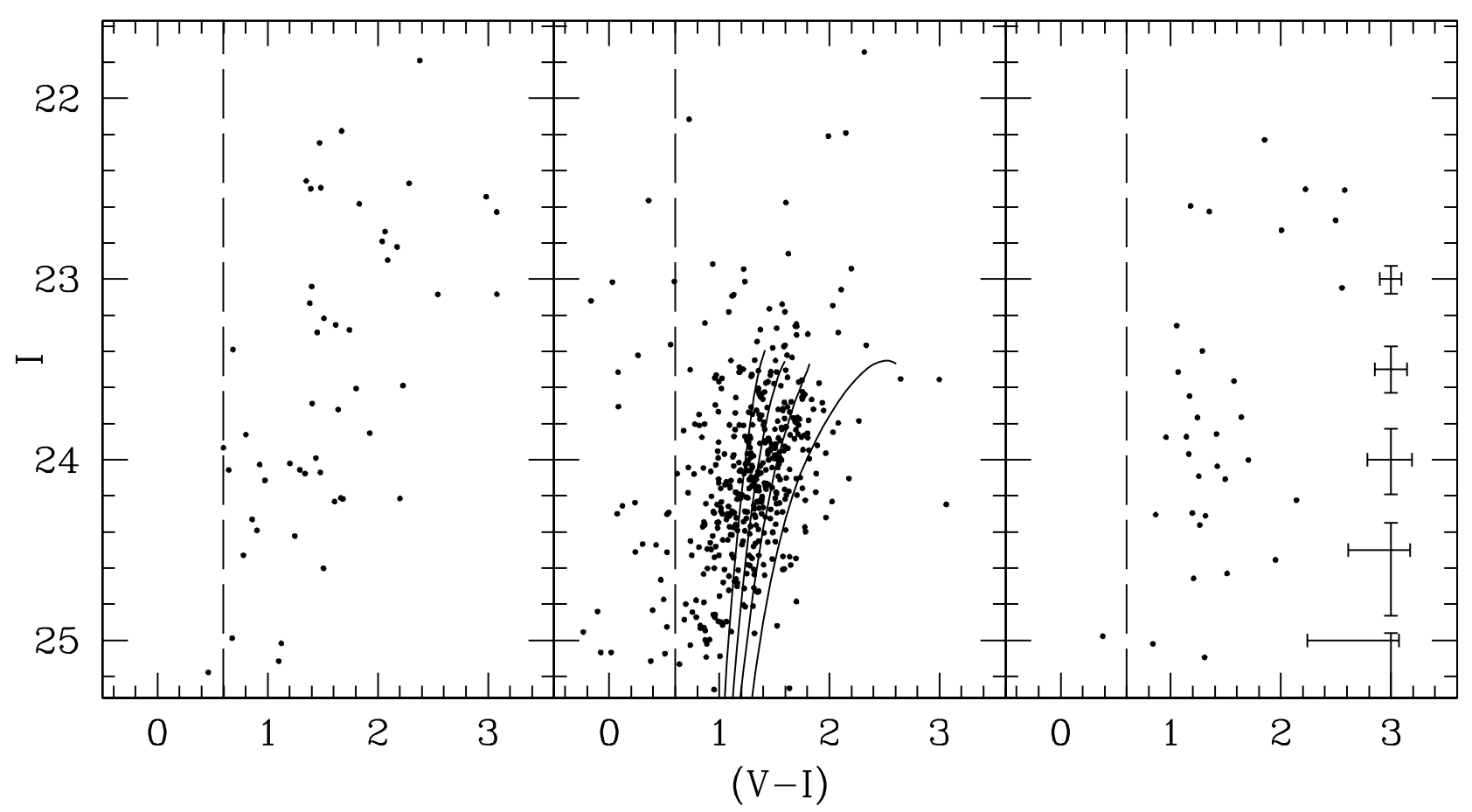

Fig. 6. The central panel shows the $I$-band colour-magnitude diagram of all stars matched in $V$ and $I$-band images and within the 40 arcsec radius from the galaxy center. Solid lines from blue to red represent the standard globular cluster branches for M $15([\mathrm{Fe} / \mathrm{H}]=-2.17), \mathrm{M} 2([\mathrm{Fe} / \mathrm{H}]=-1.58)$, NGC $1851([\mathrm{Fe} / \mathrm{H}]=-1.16)$, and $47 \mathrm{Tuc}([\mathrm{Fe} / \mathrm{H}]=-0.71)$ taken from Da Costa \& Armandroff (1990) and shifted to the distance modulus and reddening of ESO 540-032. For comparison we show in the left and right panel the CMDs for two comparison fields produced from an equivalent area on the CCD. To illustrate the presence of a significant blue stellar population in the galaxy, a dashed line is drawn at $(V-I)=0.6$ in each panel. The error bars reflect the photometric uncertainties and systematic errors

distance. The standard strategy is to determine the apparent magnitude of the tip which appears as a prominent local feature in the $I$-band luminosity function. For that purpose, the observed and completerness-corrected luminosity functions of all stars with $V-I>0.9$ (Fig. 8) are convolved with a zero-sum Sobel kernel $[-2,0,2]$. The resulting two edge-detection functions (Fig. 8) reach their maximum at the same magnitude, where the count discontinuity is the greatest. This technique of locating the tip in an objective manner was tested by Lee et al. (1993). It provides consistent results independently of the chosen bin size or photometric precision.

As the TRGB magnitude we use the midpoint of the corresponding magnitude bin $I_{\mathrm{TRGB}}=23.48 \pm 0.09 \mathrm{mag}$, where the error estimate is the combination of the width of the spike centered on the apparent magnitude of the TRGB $( \pm 0.05 \mathrm{mag})$ and the zero-point uncertainty of the photometry $( \pm 0.07 \mathrm{mag})$. Adopting $M_{I}=-4.2 \pm 0.1 \mathrm{mag}$ as the absolute magnitude and the reddening value $A_{I}=$ $0.040 \pm 0.006$ from Schlegel et al. (1998), we obtain for ESO 540-032 a distance modulus of $(m-M)_{0, \mathrm{TRGB}}=$ $27.64 \pm 0.14 \mathrm{mag}(3.4 \pm 0.2 \mathrm{Mpc})$.

In principle, the start of the steep rise in the luminosity function could also be the consequence of a numerous AGB population. The RGB tip would be roughly one magnitude fainter at $I \approx 24.48$ where a clear detection is prevented by incompleteness. To test this possibility we assumed that the stars in the magnitude range
$23.48<I<24.48$ are AGB stars. We then adopted a number-scaled version of the $I$-band luminosity function of Phoenix (Martínez-Delgado et al. 1999) to estimate the total light from all stars in ESO540-032. The resulting magnitude is more than 0.5 mag brighter than the true value as derived in Sect. 6 based on aperture photometry. Therefore the scenario of a large AGB population can be ruled out.

The distance of 3.4 Mpc confirms ESO 540-032 as a member of the nearby, sparsely populated and complicate aggregate of galaxies we know as the Sculptor group. The elongated galaxy distribution of this group has a substantial depth, with member galaxies like NGC 55, ESO 294010 , and ESO 410-G005 at the near side at $\approx 1.7 \mathrm{Mpc}$, and NGC 45 and NGC 59 at the far side at $\approx 4.4$ Mpc (JFB98; Karachentsev et al. 2000). In the sky, ESO 540-032 is close to the Sc galaxy NGC 247. However, their line-of-sight distances differ by $0.9 \mathrm{Mpc}$ which puts ESO 540-032 right in the field behind the spiral where the two dwarf irregulars DDO 006 (UGCA 015) and DDO 226 (IC 1574) were found (Côté et al. 1997).

\section{Comparison with surface brightness fluctuation distance}

The derived TRGB distance modulus for ESO 540-032 is significantly larger than the previous estimate based on the SBF method at $(m-M)_{0, \mathrm{SBF}}=26.72 \pm 0.13 \mathrm{mag}$ or 

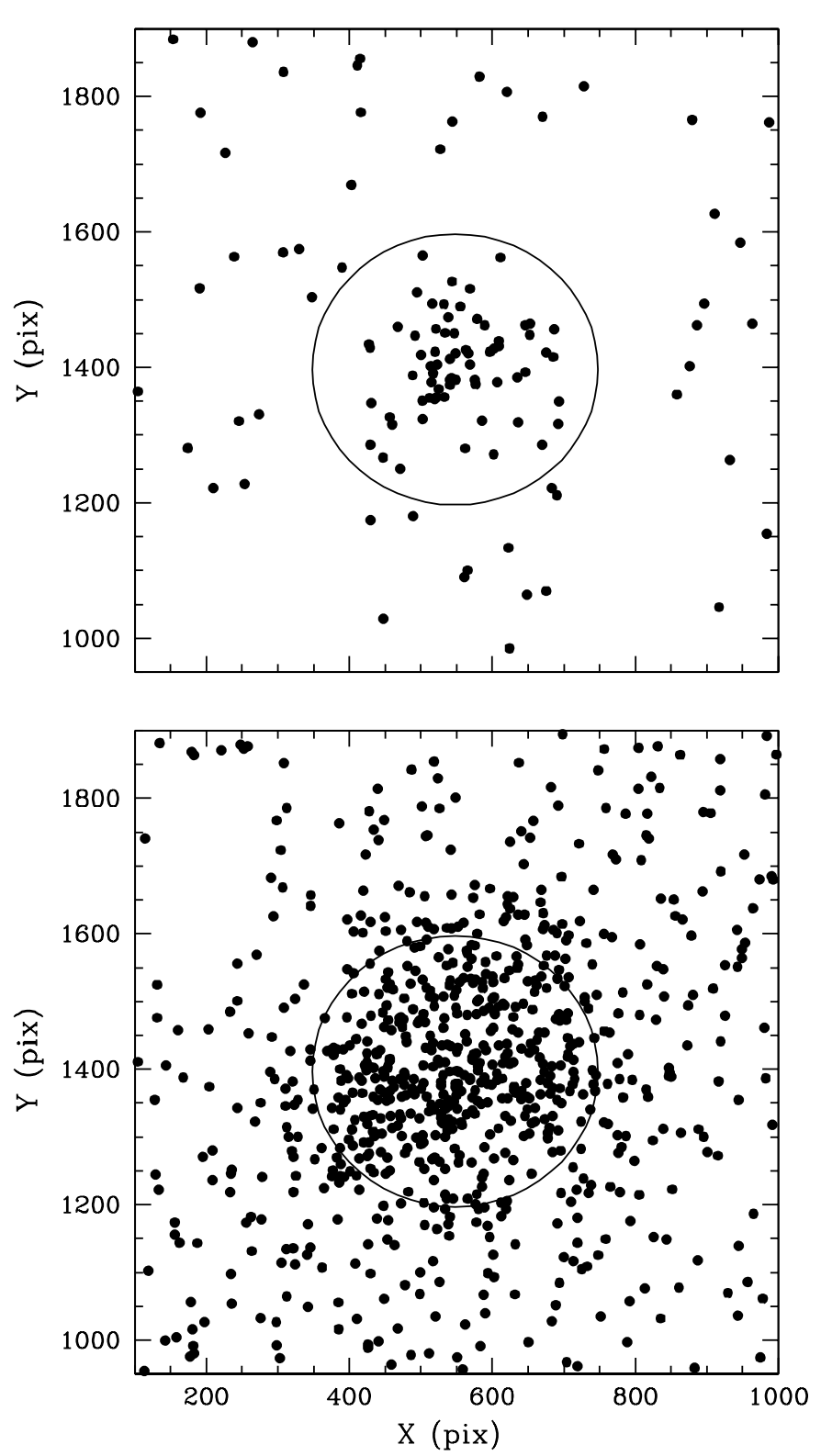

Fig. 7. Pixel maps of all detected stars in the galaxy and its vicinity. The circles are indicating the 40 arcsec boundary $\left(\mu_{I} \approx 25.0 \mathrm{mag} \operatorname{arcsec}^{-2}\right)$ of the galaxy. Stars bluer than $(V-I)=0.9$ (top panel) show a higher concentration to the center than the red stars (bottom panel)

$2.2 \pm 0.14 \mathrm{Mpc}$ (JFB98). In the latter study, the apparent fluctuation $R$-band magnitude $\bar{m}_{R}$ was recovered from the power spectrum of the galaxy image and converted into a distance modulus using predictions for $\bar{M}_{R}$ based on Worthey's (1994) stellar population synthesis models combined with Yale isochrones. Thereby, the underlying stellar population was assumed to be mainly old $(8.5 \mathrm{Gyr}<$ age $<13 \mathrm{Gyr})$ and metal-poor $(-1.9<[\mathrm{Fe} / \mathrm{H}]<-1.2)$ with a small pollution, up to the $10 \%$ level in mass, of young (5 Gyr), solar-metallicity stars. The modelling provided consistent results for such mixtures with $\bar{M}_{R}=$ $-1.13 \pm 0.06$ mag. However, the one question remained

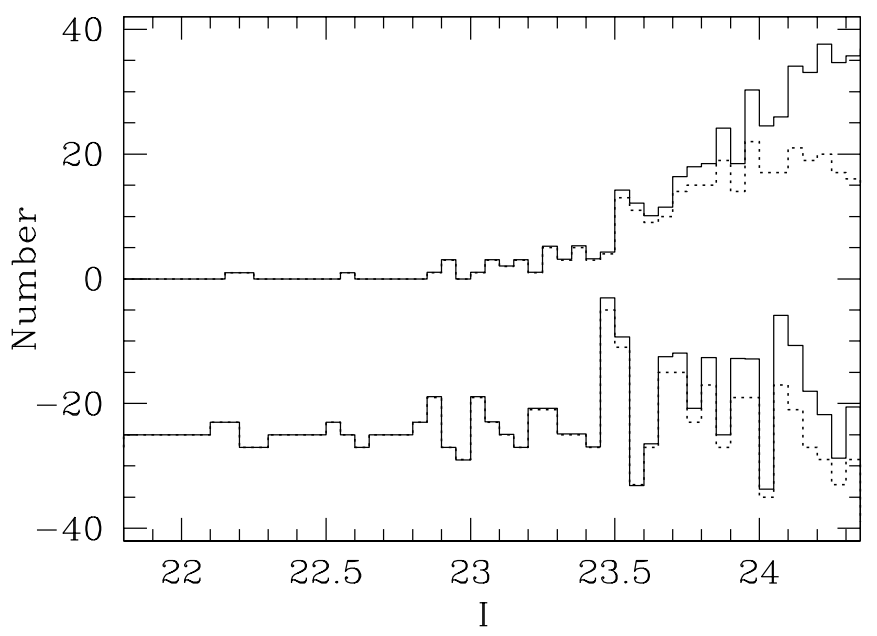

Fig. 8. The top histograms show the observed (dashed line) and completeness-corrected (solid line) I-band luminosity functions for the red giant stars $(V-I>0.9)$ in ESO 540-032. The convolutions of the luminosity functions with the Sobel edge-detection filter are the two corresponding bottom lines. The maxima of these edge-detector functions occur at the same magnitude $I \approx 23.5 \mathrm{mag}$ and mark the steepest rise in the luminosity functions, i.e. the tip of the red giant branch. The bin size is $0.05 \mathrm{mag}$

about the influence of a young ( $<8 \mathrm{Gyr})$ but metal-poor $([\mathrm{Fe} / \mathrm{H}]<-0.225)$ polluting stellar population on the SBF zero point. As JFB98 pointed out, that part of the parameter space was not accepted by the model program.

In the meantime, Worthey incorporated the isochrones from the Padova library (Bertelli et al. 1994) in his program $^{2}$ which not only yields significantly better predictions for $\bar{M}_{R}$ (for a discussion see Jerjen et al. 2000a) but also allows to test the still unexplored parameter space. Indeed, the results are quite different there. If for instance an old, metal-poor population $(12 \mathrm{Gyr},[\mathrm{Fe} / \mathrm{H}]=-1.7)$ is mixed ( $2 \%$ in mass) with a young, slightly more metal-rich population (500 Myr, $[\mathrm{Fe} / \mathrm{H}]=-1.3)$, Worthey's models give $\bar{M}_{R}=-2.37$ with a colour of $(B-R)_{0}=0.98$. Using the newly derived zero point to recalibrate the fluctuation signals measured for the two fields F1 and F2 which have been analysed in JFB98 and having estimated colours of $(B-R)_{0, \mathrm{~F} 1}=0.89$ and $(B-R)_{0, \mathrm{~F} 2}=0.99$, we compute a distance modulus of $27.93 \pm 0.15$. This value now is in better agreement with the result from the TRGB analysis.

Despite the apparent resolution of the distance discrepancy in the case of ESO 540-032, we are not proposing to extend the application of the SBF technique to intermediate-type dwarfs. The value of $\bar{M}_{R}$ turns out to be highly variable for such systems. It depends sensitively on the age and metallicity of the young stars as well as on the mass ratio $\mathcal{M}_{\text {young }} / \mathcal{M}_{\text {old }}$ of the two subpopulations. The SBF is best measured in dwarf ellipticals and dwarf S0s, i.e. in dwarfs that show essentially no star formation subsequent to an initial formation episode. It seems very

\footnotetext{
${ }^{2}$ http://199.120.161.183:80/ worthey/dial/dial_a_ model.html
} 
difficult if not impossible to predict the exact SBF zero point for an intermediate-type dwarf without profound knowledge of its stellar content. Thus, to obtain an accurate SBF distance would imply the analysis of a CMD which would naturally lead to a distance via a TRGB measurement.

The found discrepancy between the TRGB and SBF results for ESO 540-032 suggests the SBF distances for the two other intermediate-type Scl group dwarf galaxies ESO 294-010 and ESO 540-030 are too small as well. While the location of ESO 294-010 at the near side of the Scl group seems to be confirmed by the low redshift at $v_{\odot}=$ $117 \pm 5 \mathrm{~km} \mathrm{~s}^{-1}$ (JFB98), additional work will be needed to get a final answer in the case of ESO 540-030.

\section{Galaxy light profiles and model parameters}

We cleaned the VI galaxy images from foreground stars and background galaxies using procedures written within the IRAF package. Contaminating objects in the vicinity of the galaxy were replaced by patches of plain sky. If an object affected the galaxy light, the area was replaced instead by a nearby uncontaminated patch from the same surface brightness range. The coordinates of the center of the luminosity-weighted light distribution was adopted as the galaxy center. Using the IRAF command ellipse, the mean ellipticity of the galaxy was measured to be $e=0.20$. We performed simulated aperture photometry on the clean images to produce growth curves and $V I$ surface brightness profiles (Fig. 9) as a function of the semi-major axis. The growth curve that converges best to a plateau at large distances from the galaxy determined the total magnitude $m_{\mathrm{T}}$ and the magnitude uncertainty was estimated by varying the sky brightness. At half of the asymptotic intensity we read off the half-light ("effective") radius, $r_{\text {eff }}$, and calculated the mean surface brightness within $r_{\text {eff }}$ : the "effective surface brightness" $\langle\mu\rangle_{\text {eff }}$. All photometric and structure parameters for ESO 540032 are listed in Table 3.

The observed surface brightness profiles clearly deviate from an exponential light profile (a straight line) showing a continuous flattening towards the center. Thus, generalized exponential functions (Sérsic 1968): $I(r)=$ $I_{0} \exp \left[-\left(r / r_{0}\right)^{n}\right]$, with a free shape parameter $n$, were fitted to the data. The inner and outer cut-offs for the fits were chosen to be at 3 and 80 arcsec, respectively. The best-fitting Sérsic profiles are shown as solid lines in Fig. 9 and the corresponding model parameters, i.e. the scale length $r_{0}$, the central surface brightness $\mu_{0}$, and the shape parameter $n$ listed in Table 3 . The overall errors quoted include the profile fitting error and the error due to the uncertainty in the sky determination.

The shape parameters in $V$ and $I$ are consistent with those measured in other passbands $\left(n_{B}=1.21 \pm 0.07\right.$ and $n_{R}=1.36 \pm 0.03$, Jerjen et al. 2000b). The scale length $r_{0}$ increases slightly to redder passbands from $r_{B, 0}=19.9$ arcsec to $r_{I, 0}=24.6$ arcsec which can be explained by

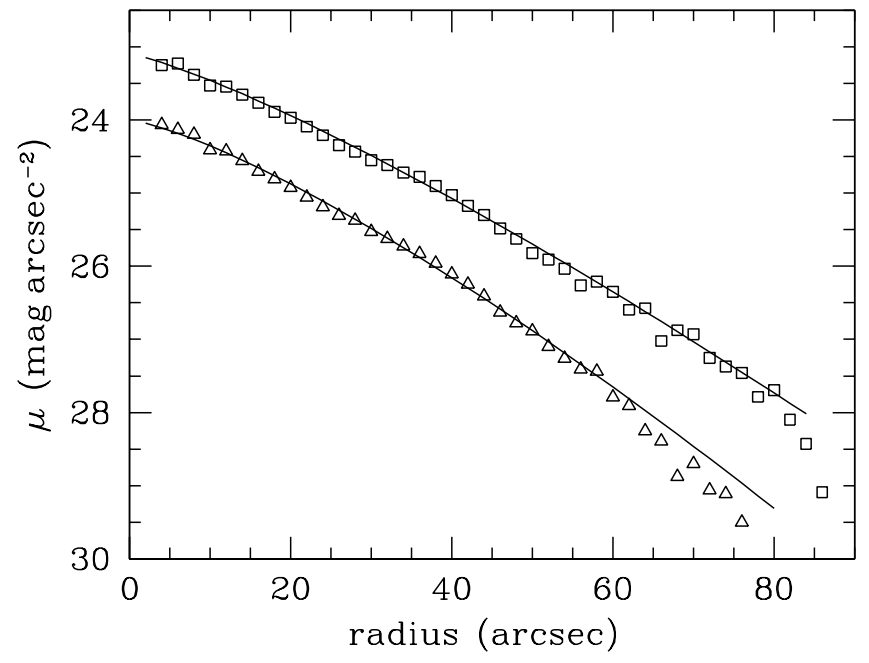

Fig. 9. Radial $V$ (triangles) and $I$-band (boxes) surface brightness profiles of ESO 540-032. The solid lines are the best-fit Sérsic profiles using the model parameters in Table 3

the high concentration of blue stars in the galaxy center (Sect. 3).

From the TRGB distance, we derive a dereddened absolute $V$ magnitude $M_{V}=-12.1 \mathrm{mag}$ and a $V$ central surface brightness of $24 \mathrm{mag} \operatorname{arcsec}^{-2}$. If plotted versus the galaxy's mean metallicity $-1.7 \pm 0.3 \mathrm{dex}$, these data points further support the two relations between metallicity and $M_{V}$ and metallicity and central surface brightness defined by the dwarf elliptical galaxies in the Local Group (Caldwell et al. 1998; Caldwell 1999).

\section{Globular cluster candidate}

While reducing the photometric data, we noticed a nonstellar object at the north eastern part of the galaxy $\left(00^{\mathrm{h}} 50^{\mathrm{m}} 24.1^{\mathrm{s}},-19^{\circ} 54^{\prime} 15^{\prime \prime}, \mathrm{J} 2000\right), 11^{\prime \prime} .2$ from the center (see Fig. 10). It has a profile that is more globular cluster (GC) like in the sense that the light distribution has broad wings with a dispersion $\sigma=1.18$ arcsec. The mean $F W H M$ of a star on the CCD frame is $\sim 0.6$ arcsec. If this object is indeed a GC, we get a rough estimate of its core radius $r_{\mathrm{c}}$ with the form $2 r_{\mathrm{c}}=\sqrt{\sigma_{\mathrm{GC}}^{2}-\sigma_{\mathrm{star}}^{2}}=$ 1.02 arcsec. Employing our TRGB distance this translates into $r_{\mathrm{c}}=8.4 \mathrm{pc}$. For comparison, the core radius of $\omega$ Cen, the most luminous GC in the Milky Way has only a core radius of $3.8 \mathrm{pc}$ (Harris 1996). However, there are several Galactic GCs that have core radii even larger than the one we estimate from our images (e.g. NGC 5053, Pal5, Pal14, Pal145, Pyxis; see Harris' web catalogue $\left.{ }^{3}\right) .(V-I)_{0}$ colour as well as $V$ and $I$ magnitudes of the extended object $\left[(V-I)_{0}=0.9, V_{0}=-7.5\right.$ and $\left.I_{0}=-8.4 \mathrm{mag}\right]$ are within the range observed for the MW globular clusters (Harris 1996).

Alternatively, the unknown object could well be an H II region like the one identified in ESO 294-010 (JFB98) or a

\footnotetext{
3 http://physun.physics.mcmaster.ca/Globular.html
} 
Table 3. Properties of ESO 540-032

\begin{tabular}{|c|c|}
\hline Type & $\mathrm{dE} / \operatorname{Im}(\mathrm{Sph} / \mathrm{dIrr})$ \\
\hline RA (J2000.0) & $00^{\mathrm{h}} 50^{\mathrm{m}} 24.6^{\mathrm{s}}$ \\
\hline Decl. (J2000.0) & $-19^{\circ} 54^{\prime} 23^{\prime \prime}$ \\
\hline Galactic $l$ (deg) & 121.00962 \\
\hline Galactic $b(\mathrm{deg})$ & -82.77422 \\
\hline SuperGalactic $l$ (deg) & 276.95088 \\
\hline SuperGalactic $b$ (deg) & -4.24336 \\
\hline$e$ & 0.20 \\
\hline$V_{\mathrm{T}}(\mathrm{mag})$ & $15.58 \pm 0.12$ \\
\hline$I_{\mathrm{T}}(\mathrm{mag})$ & $14.55 \pm 0.17$ \\
\hline$E(B-V)(\mathrm{mag})$ & 0.020 \\
\hline Extinction: $A_{V}, A_{I}(\mathrm{mag})$ & $0.068,0.040$ \\
\hline$r_{V, \mathrm{eff}}(\operatorname{arcsec})$ & $27.3 \pm 1.8$ \\
\hline$\langle\mu\rangle_{V, \text { eff }}\left(\operatorname{mag} \operatorname{arcsec}^{-2}\right)$ & $24.8 \pm 0.08$ \\
\hline$r_{I, \mathrm{eff}}(\operatorname{arcsec})$ & $29.4 \pm 2.2$ \\
\hline$\langle\mu\rangle_{I, \mathrm{eff}}\left(\mathrm{mag} \operatorname{arcsec}^{-2}\right)$ & $23.9 \pm 0.09$ \\
\hline$\mu_{V, 0}\left(\operatorname{mag} \operatorname{arcsec}^{-2}\right)$ & $24.0 \pm 0.2$ \\
\hline$r_{V, 0}(\operatorname{arcsec})$ & $23.6 \pm 2.7$ \\
\hline$n_{V}$ & $1.30 \pm 0.12$ \\
\hline$\mu_{I, 0}\left(\mathrm{mag} \operatorname{arcsec}^{-2}\right)$ & $23.1 \pm 0.1$ \\
\hline$r_{I, 0}(\operatorname{arcsec})$ & $24.6 \pm 1.6$ \\
\hline$n_{I}$ & $1.23 \pm 0.06$ \\
\hline$I_{\mathrm{TRGB}}(\mathrm{mag})$ & $23.48 \pm 0.09$ \\
\hline$(m-M)_{0}(\mathrm{mag})$ & $27.64 \pm 0.14$ \\
\hline Distance (Mpc) & $3.4 \pm 0.2$ \\
\hline$\langle[\mathrm{Fe} / \mathrm{H}]\rangle(\mathrm{dex})$ & $-1.7 \pm 0.3$ \\
\hline
\end{tabular}

galaxy located in the background of ESO 540-032 similar to the case of BK5N, a dwarf in the M 81 group, where an apparent nucleus was in fact a distant spiral galaxy (Caldwell et al. 1998).

\section{Summary and conclusions}

Based on CCD images obtained at the ESO Very Large Telescope UT1+FORS1, we presented the $(I, V-I)$ colour-magnitude diagram and the $I$-band luminosity function for the nearby dwarf galaxy ESO 540-032. The CMD shows traces of two distinct stellar components, a predominant population of metal-poor $([\mathrm{Fe} / \mathrm{H}] \approx$ -1.7 dex) stars of ages similar to those of globular clusters and a small fraction of young (150-500 Myr), more metal-rich $([\mathrm{Fe} / \mathrm{H}] \approx-1.3 \mathrm{dex})$ stars. In addition, the CMD reveals a considerable number of stars brighter than the RGB tip. They could be AGB stars and represent an intermediate-age population. In this case, ESO 540-032 would have had a continuous star formation activity like the Fornax dwarf. Alternatively, these stars are foreground stars, a scenario which is supported by the CMDs of comparison fields and thus more likely. The young stars in ESO 540-032 would then be the result of a single event

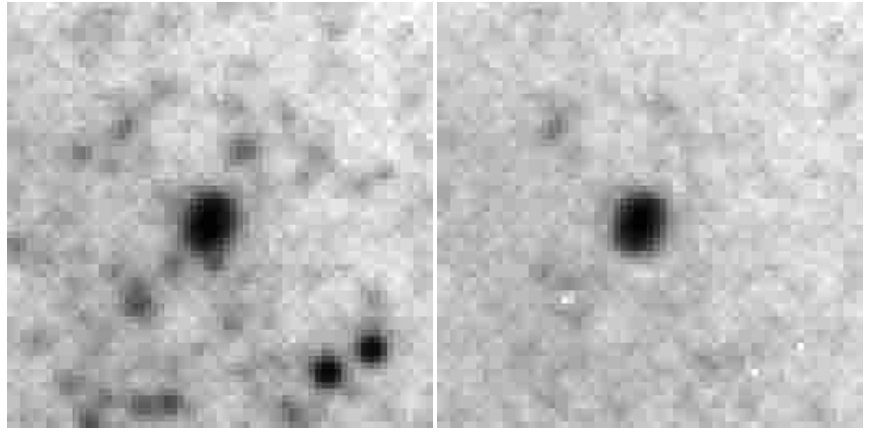

Fig. 10. The $V$ image of the globular cluster candidate before (left) and after (right) the subtraction of neighbouring stars. The cleaned image was produced by the allstar task in IRAF using the stellar PSF. The size of the images is $12^{\prime \prime} .8 \times 12^{\prime \prime} .8$. North is up, east to the left

comparable to the situation observed in the two Local Group dwarfs Phoenix and LSG3.

The tip of the red giant branch magnitude $I_{0}=$ $23.48 \pm 0.09$ mag derived from the $I$-band luminosity function of ESO 540-032 corresponds to a true distance modulus of $27.64 \pm 0.14 \mathrm{mag}$, or $D=3.4 \pm 0.2 \mathrm{Mpc}$. This new distance for ESO 540-032 seems to be more reliable than the previously published value of $D=2.2 \pm 0.14 \mathrm{Mpc}$. The latter result was based on a successful measurement of the surface brightness fluctuation signal in the galaxy but relied on incorrect assumptions about the stellar mixture. From our experiments with Worthey's stellar population synthesis models, we draw the conclusion that there is no advantage for the SBF method over the TRGB method to measure distances of intermediate-type $(\mathrm{dE} / \mathrm{Im})$ dwarf galaxies. Applications of the SBF technique should be concentrated on genuine dwarf elliptical and dwarf S0 galaxies.

In space, ESO 540-032 is located in the field behind the spiral galaxy NGC 247, a region which is part of the elongated nearby cloud of galaxies in Sculptor. Its VI surface brightness profiles have bee derived and fitted by Sérsic models. The mean metallicity, the total $V$ luminosity and the central surface brightness in $V$ follow closely the relations exhibit by the early-type dwarf galaxies in the Local Group. In ESO 540-032, we are likely to witness the final stage of the transition from a dwarf irregular to a dwarf elliptical galaxy. Finally, we discussed the properties of an extended object in the central area of ESO 540-032. Colour, physical size, and absolute magnitudes do not rule out that it is a large globular cluster what we see.

The first priority of future work will be to measure colour-magnitude diagrams for the remaining four dwarfs NGC 59, Scl-dE1, ESO 294-010, and ESO 540-030 of the JFB98 sample in the Sculptor group region. They will allow to determine metallicities and metallicity spreads and to search for evidence of recent star formation activities from blue-loop stars. The tip of the red giant branch distances will provide a check of the existing SBF distances. If the data are combined with radial velocities, these dwarfs can be used as additional test particles for dynamical 
models of the Sculptor-Local Group cloud which will help to refine our knowledge of this complex structure in the Supergalactic plane.

Acknowledgements. We are grateful to the anonymous ESO service observer at the VLT UT1 (Antu) for providing excellent quality images for this study. It is a pleasure to thank G. Da Costa and B. Binggeli for useful discussions and the referee G. P. Bertelli for interesting comments. HJ acknowledges the financial support from the Swiss National Science Foundation.

\section{References}

Aparicio, A., Gallart, C., \& Bertelli, G. 1997, AJ, 114, 680

Bertelli, G., Bessan, A., Chiosi, C., Fagotto, F., \& Nasi, E. 1994, A\&AS, 106, 271

Caldwell, N. 1999, AJ, 118, 1230

Caldwell, N., Armandroff, T. E., Da Costa, G. S., \& Seitzer, P. 1998, AJ, 115, 535

Côté, S. 1995, Ph.D. Thesis, Australian National University

Côté, S., Freeman, K. C., Carignan, C., \& Quinn, P. J. 1997, AJ, 114, 1313

Da Costa, G. S. 1998, in Stellar astrophysics for the local group, VIII Canary Islands Winter School of Astrophysics, ed. A. Aparicio, A. Herrero, \& F. Sanchez (Cambridge, New York, Cambridge University Press), 351

Da Costa, G. S., \& Armandroff, T. E. 1990, AJ, 100, 162

Gallagher, III, J. S., Littleton, J. E., \& Matthews, L. D. 1995, AJ, 109, 2003

Graham, J. A. 1981, PASP, 93, 29

Gratton, R. G., Fusi Pecci, F., Carretta, E., et al. 1997, ApJ, 491, 749

Grebel, E. K. 1998, in IAU Symp. 192, The Stellar Content of Local Group Galaxies, ed. P. Whitelock, \& R. Cannon
(San Francisco: ASP), 1

Harris, W. E. 1996, AJ, 112, 1487

Held, E. V., Saviane, I., \& Momany, Y. 1999, A\&A, 345, 747

Holtzman, J. A., Smith, G. H., \& Grillmair, C. 2000, AJ, 120, 3060

Hurley-Keller, D., Mateo, M., \& Nemec, J. 1998, AJ, 115, 1840

Jerjen, H., Freeman, K. C., \& Binggeli, B. 1998, AJ, 116, 2873

Jerjen, H., Binggeli, B., \& Freeman, K. C. 2000b, AJ, 119 , 593

Jerjen, H., Freeman, K. C., \& Binggeli, B. 2000a, AJ, 119, 166

Karachentseva, V. E., \& Karachentsev, I. D. 1998, A\&AS, 127, 409

Karachentsev, I. D., Makarov, D. I., \& Huchtmeier, W. K. 1999, A\&AS, 139, 97

Karachentsev, I. D., Sharina, E., Grebel, E., et al. 2000, ApJ, 542,128

Landolt, A. U. 1992, AJ, 104, 340

Lee, M.-G., Freedman, L. W., \& Madore, B. F. 1993, 417, 553

Martínez-Delgado, D., Gallart, C., \& Aparicio, A. 1999, AJ, 118,862

Mighell, K. J. 1997, AJ, 114, 1458

Olszewski, E. W., \& Aaronson, M. 1985, AJ, 90, 221

Robin, A. C., Haywood, M., Creze, M., Ojha, D. K., \& Bienayme, O. 1996, A\&A, 305, 125

Salaris, M., \& Cassisi, S. 1998, MNRAS, 298, 166

Schlegel, D., Finkbeiner, D., \& Davis, M. 1998, ApJ, 500, 525

Sérsic, J.-L. 1968, Atlas de galaxias australes, (Cordoba: Observatorio Astronomico)

Stetson, P. B. 1987, PASP, 99, 191

Stetson, P. B., Hesser, J., \& Smecker-Hane, T. E. 1998, PASP, 110,533

St-Germain, J., Carignan, C., Côté, S., \& Oosterloo, T. 1999, AJ, 118, 1235

Walker, A. R. 1995, PASP, 107, 683

Worthey, G. 1994, ApJS, 95, 107 